\title{
Beatmungsassoziierte Pneumonie - Prävention und Diagnostik
}

\section{K.-F. Bodmann}

\section{Ventilator-associated pneumonia - prevention and diagnostic}

\section{Pråvention und Diagnostilk}

Nosokomiale Pneumonien stellen die zweithäufigste nosokomiale Infektion in den westlichen Industrieländern dar. Wie aus der NIDEP(Nosokomiale Infektionen in Deutschland - Erfassung und Prävention)-Studie (1) zu entnehmen ist, beträgt die Prävalenz nosokomialer Infektionen etwa 4\%. Die unteren Atemwegsinfektionen lagen wie im angelsächsischen Schrifttum mit 20,6\% an der zweiten Stelle. Darunter entfielen 75\% auf Pneumonien. In der Intensivmedizin wurde die höchste Prävalenz der unteren Atemwegsinfektionen im Krankenhaus ermittelt; ihr Anteil an allen nosokomialen Infektionen betrug $53,4 \%$. Dies ist vor allem deshalb von Bedeutung, weil Infektionen die führende Todesursache bei Todesfällen mit Bezug zum Krankenhaus sind.

Der wichtigste Risikofaktor für nosokomiale Pneumonien ist die maschinelle Beatmung mit endotrachealer Intubation; bei beatmeten Patienten ist das Risiko bis zu 21-mal höher als bei anderen Patienten. Unter maschineller Beatmung steigt das kumulative Risiko, an einer nosokomialen Pneumonie zu erkranken, proportional zur Beatmungsdauer. Die kumulative Inzidenz der Ergebnisse des Krankenhaus-Infektions-Surveillance-Systems (KISS) $(2,3)$ lassen einen noch höheren Stellenwert der nosokomialen Pneumonie erkennen. Sie stellt hier die häufigste nosokomiale Infektion dar. Der größte Teil aller Infektionen war mit dem Gebrauch von intrakorporalen Fremdkörpern assoziiert. So traten $89 \%$ aller nosokomialen Pneumonien bei intubierten und maschinell beatmeten Patienten auf.

Während einer Langzeitbeatmung (länger als 10 Tage) entwickelt sich bei mehr als 75\% der Erkrankten eine Pneumonie (4). Hierbei spielen das Vorliegen einer strukturellen Lungenerkrankung und eine vorbestehende Antibiotikatherapie, die zu einer Selektion multiresistenter Erreger führt, eine wichtige Rolle.

\section{Pråvention}

Zur Vermeidung nosokomialer Pneumonien unter Respiratortherapie muss ein schlüssiges Hygienekonzept vorliegen. Die Einhaltung dieses Präventionskonzeptes bedarf einer strengen Kontrolle. Auch die Erfassung nosokomialer Pneumonien sollte mittels eines diagnostischen Konzeptes festgelegt werden. Seit dem 1.1.2001 ist die fortlaufende Erfassung und Dokumentation nosokomialer Infektionen durch den $§ 23$ des Infektionsschutzgesetzes vorgeschrieben. Die wichtigsten Präventionsmaßnahmen umfassen Angaben zur Intubation, und Pflege der beatmeten Patienten. Außerdem gibt es ergänzende Empfehlungen für beatmete Patienten (5) (Tab.1 u. Tab.2).

\section{Intubation}

Es muss immer eine strenge Indikation zur Intubation bestehen und es muss die schnellstmögliche Extubation angestrebt werden. Weiterhin sollte, wenn möglich, eine nicht-invasive Beatmung in Betracht gezogen werden. In Bezug auf den Intubationsweg besteht kein eindeutiger Vorteil für eine orale Intubation gegenüber einer nasalen Intubation. Bei der oralen Intubation treten jedoch weniger Sinusitiden auf.

Eine Tracheotomie und der Wechsel des Tracheotomietubus sollten unter aseptischen Bedingungen erfolgen. Die Auswahl des Trachealtubus betreffend scheint die Verwendung von Tuben mit der Möglichkeit zur kontinuierlichen subglottischen Absaugung die Anzahl nosokomialer Pneumonien reduzieren zu können. 
Tab. 1 Maßnahmen zur Prävention der beatmungsassoziierten Pneumonie.

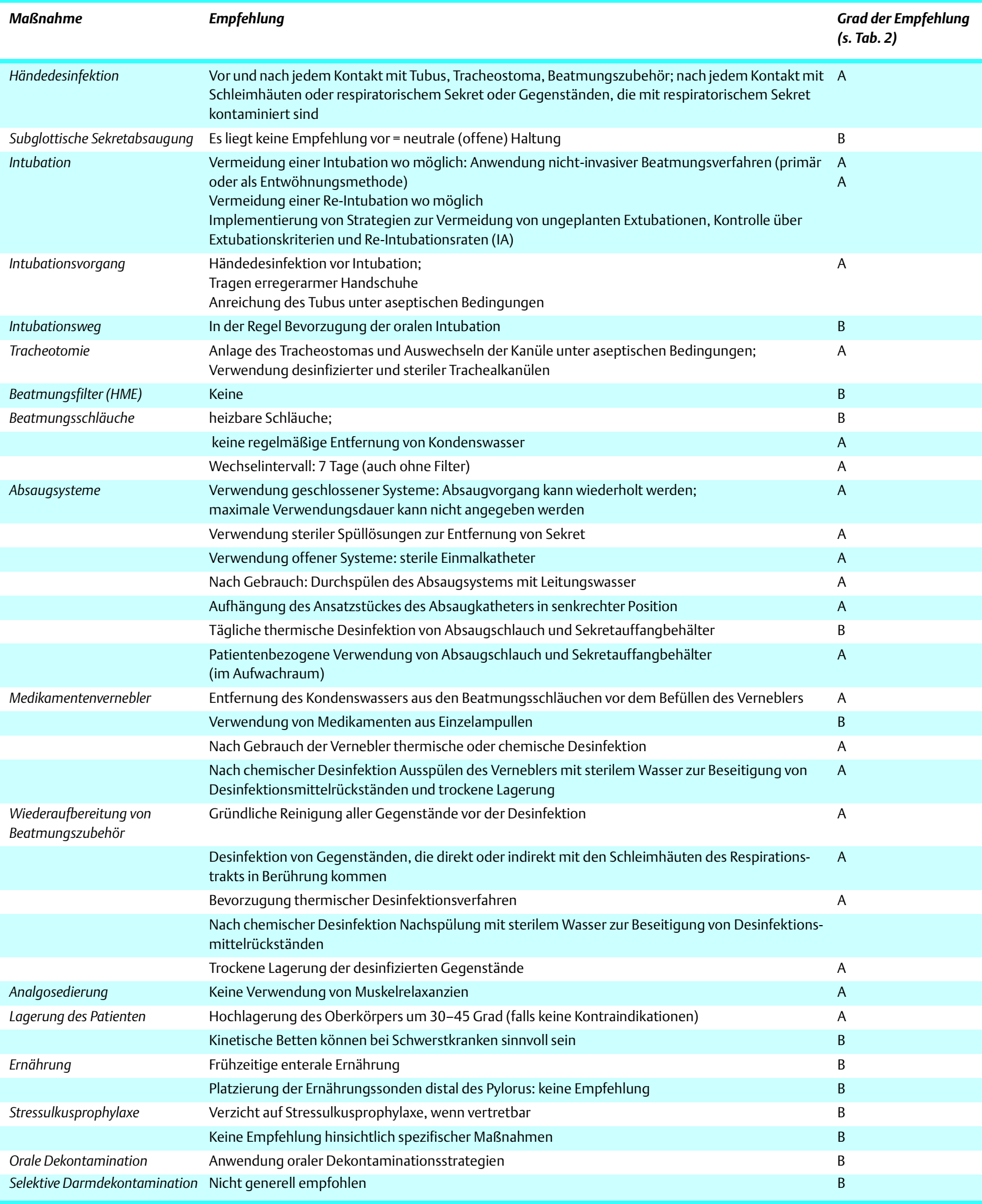


Tab. 2 Bewertung der publizierten Literatur gemäß Aussagekraft nach Evidenztypen.

\begin{tabular}{|c|c|c|}
\hline $\begin{array}{l}\text { Grad der } \\
\text { Empfehlung }\end{array}$ & Evidenz & \\
\hline A & la & $\begin{array}{l}\text { Evidenz aufgrund von Metaanalysen randomisier- } \\
\text { ter, kontrollierter Studien }\end{array}$ \\
\hline A & $\mathrm{lb}$ & $\begin{array}{l}\text { Evidenz aufgrund mindestens einer randomisier- } \\
\text { ten, kontrollierten Studie }\end{array}$ \\
\hline$B$ & Ila & $\begin{array}{l}\text { Evidenz aufgrund mindestens einer gut angeleg- } \\
\text { ten kontrollierten Studie ohne Randomisierung }\end{array}$ \\
\hline B & Ilb & $\begin{array}{l}\text { Evidenz aufgrund mindestens einer gut } \\
\text { angelegten quasi experimentellen Studie }\end{array}$ \\
\hline B & III & $\begin{array}{l}\text { Evidenz aufgrund gut angelegter nicht experimen- } \\
\text { teller deskriptiver Studien (z.B. Vergleichsstudien, } \\
\text { Korrelationsstudien, Fall-Kontrollstudien) }\end{array}$ \\
\hline$C$ & IV & $\begin{array}{l}\text { Evidenz aufgrund von Berichten/Meinungen von } \\
\text { Expertenkreisen, Konsensus-Konferenzen und/oder } \\
\text { klinischer Erfahrungen anerkannter Autoritäten }\end{array}$ \\
\hline
\end{tabular}

\section{Pflege des beatmeten Patienten}

Vor allen Manipulationen am Absaugkatheter und Beatmungssystem muss eine Händedesinfektion durchgeführt werden. Es kann keine Bevorzugung von geschlossenen Mehrfachabsaugsystemen gegenüber offenen Einmalabsaugsystemen empfohlen werden. Die Beatmungsschläuche müssen nur einmal wöchentlich gewechselt werden.

\section{Allgemeine Empfehlungen bei beatmeten Patienten}

Eine Oberkörperhochlagerung von 30-45 Grad wird empfohlen, wenn keine Kontraindikationen bestehen. Eine selektive Darmdekontamination (SDD) bei schwerkranken, mechanisch beatmeten Patienten wird zur Zeit nicht empfohlen. Eine neuere Metaanalyse zeigte jedoch nicht nur einen Vorteil in Hinblick auf die Pneumoniereduktion sondern auch hinsichtlich der Letalität.

kurzgefasst: Um nosokomialer Pneumonien bei beatmeten Patienten zu vermeiden, sollte die Indikation zur Intubation streng gestellt und eine schnelle Extubation angestrebt werden. Bei Manipulationen am Tubus oder am Beatmungssystem muss eine Händedesinfektion erfolgen.

\section{Diagnostik}

Die Hinweise zur Diagnostik der beatmungsassoziierten Pneumonie beziehen sich auf das Konsensuspapier der Paul-EhrlichGesellschaft (PEG) und der Deutschen Gesellschaft für Pneumologie (DGP) über Prävention, Diagnostik und Therapie der nosokomialen Pneumonie (6).

Die klinische Diagnose der nosokomialen Pneumonie unter Respiratortherapie erfolgt bei einem neuen oder persistierenden Infiltrat im Röntgenbild des Thorax plus zwei der folgenden drei Kriterien:

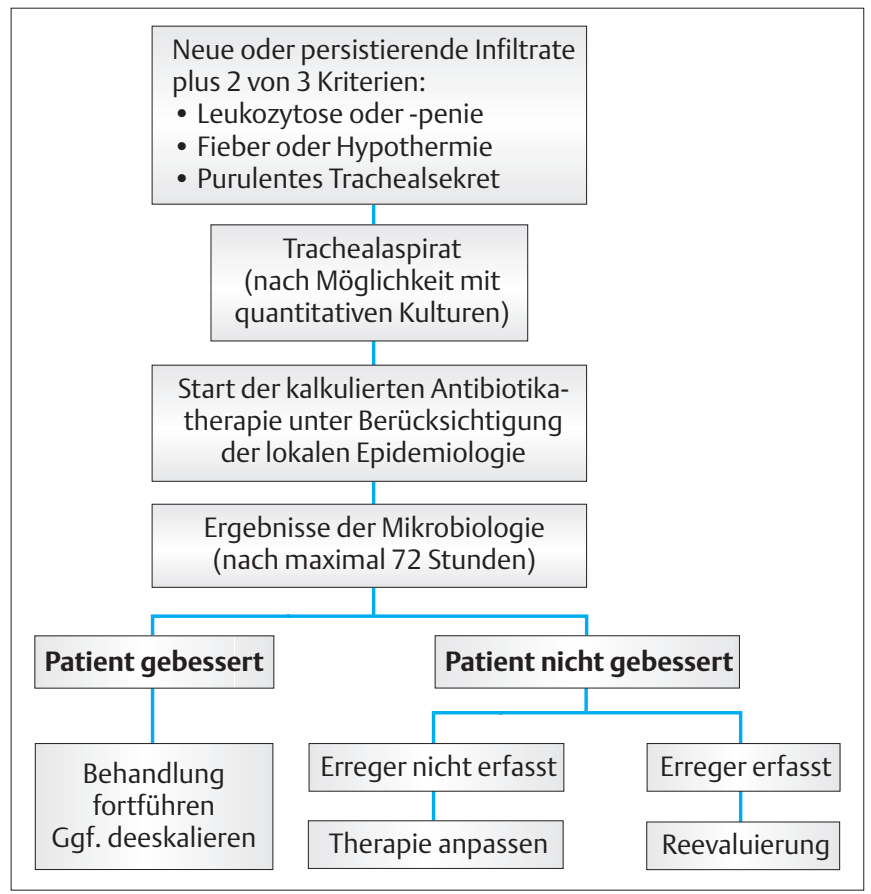

Abb. 1 Algorithmus zum empfohlenen Vorgehen bei der Diagnostik der nosokomialen Pneumonie.
1. Leukozytose (> $12 \times 10 / 1)$ oder Leukopenie $(<4 \times 10 / 1)$
2. Fieber über $38^{\circ} \mathrm{C}$ oder Hypothermie unter $36^{\circ} \mathrm{C}$
3. purulentes Bronchialsekret

Alternativ kann auch ein klinischer Score herangezogen werden, der neben den oben genannten Parametern auch Informationen wie etwa die Gasaustauschstörung einbezieht.

Zum mikrobiologischen Erregernachweis sollte die Probengewinnung vor Einleitung einer antimikrobiellen Therapie erfolgen. Falls eine antimikrobielle Therapie bereits durchgeführt wird, sollte diese 72 Stunden vor der Probengewinnung nicht umgestellt werden (7). Eine Therapiepause vor Durchführung der Diagnostik ist grundsätzlich nicht erforderlich. Transport- und Lagerungszeiten der Materialien sollten 4 Stunden nicht überschreiten, da sich ansonsten das Verhältnis von pathogenen zu nichtpathogenen Keimen verschiebt.

Sofern keine spezielle Fragestellung formuliert wird, umfasst der routinemäßige Untersuchungsumfang die mikroskopische Beurteilung der Probe mittels Gramfärbung sowie die kulturelle Untersuchung auf schnellwachsende aerobe Bakterien und Pilze. Die quantitative Kultur erhebt den Anspruch, durch einen KeimzahlTrennwert eine Unterscheidung zwischen Kolonisation und Infektion leisten zu können. Übliche Grenzen richten sich nach den angewandten diagnostischen Verfahren (geschützte Bürste $10^{3}$, BAL $10^{4}$, Trachealsekret $10^{5}$ koloniebildende Einheiten (KBE) pro $\mathrm{ml}$ Material). Der Nutzen der quantitativen Kultur ist aber umstritten. Eine hohe Keimzahl kann zwar zur Bestätigung des verantwortlichen Erregers eingesetzt werden. Im Umkehrschluss kann eine Pneumonie aber aufgrund einer unzureichenden Bakterienkonzentration nicht ausgeschlossen werden. Dies gilt insbesondere für antibiotisch vorbehandelte Patienten. Für die beatmungsassoziierte Pneumonie wurde bisher die invasive Diagnostik mit bronchoskopischer Materialentnahme (geschützte Bürs- 
te, bronchoalveoläre Lavage) als Standard erachtet und gefordert. Diese Formen der Probenentnahme bieten jedoch im Vergleich zum quantitativ untersuchten Trachealsekret keinen Vorteil im Hinblick auf das Therapieergebnis (Letalität, Beatmungsdauer und Verweildauer auf der Intensivstation) (8).

\section{Blutkulturen}

Es sollen mindestens zwei Blutkultursets (jeweils aerob und anaerob), von unterschiedlichen Punktionsstellen, abgenommen werden. Über den optimalen Zeitpunkt für die Abnahme der Blutkulturen liegen keine gesicherten Daten vor (9). Insgesamt 5-10\% der Fälle verlaufen bakteriämisch und können mit der Blutkultur aufgedeckt werden. Im Falle einer positiven Blutkultur müssen andere Infektionsquellen ausgeschlossen werden (z.B. Kontamination). Trotz niedriger Sensitivität ist eine Blutkultur obligat, da die hohe Spezifität eine gezielte antimikrobielle Therapie ermöglicht und andererseits eine positive Blutkultur eine schlechtere Prognose anzeigt.

\section{Serologie}

Die Serologie spielt für die Akutdiagnostik keine Rolle. Für epidemiologische Fragestellungen können Untersuchungen von Bedeutung sein. Die Bestimmung des Candida-Antigens im Serum hat für die Diagnostik pulmonaler Candidosen keinen klinischen Stellenwert.

\section{Ergusspunktat}

Eine Thorakozentese ist indiziert bei großem Erguss mit Dyspnoe oder Verdacht auf Empyem. Bei einem radiologisch oder sonographisch nachweisbaren Pleuraerguss kann die Punktion zu Differenzialdiagnostik indiziert sein (z.B. Verdacht auf Neoplasie). Im Pleurapunktat werden bestimmt: pH, Eiweiß, Lactatdehydrogenase (LDH), Glucose, Zytologie, Bei einem Proteinoder LDH-Quotienten Serum/Pleuraerguss >0,6 oder LDH im Erguss von>200 U/L besteht ein Exsudat). Der Ausstrich wird zusätzlich nach Gram gefärbt und gegebenenfalls auf Mykobakterien untersucht. Zusätzlich müssen Kulturen angelegt werden.

\section{Tracheobronchialsekret}

Die Kontamination durch kolonisierende Erreger wird bei intubierten Patienten gering gehalten, indem initial das Sekret im Tubus abgesaugt wird und danach ein neuer Katheter mit angeschlossenen Auffanggefäß tief eingeführt wird. Erst dann wird für diagnostische Zwecke abgesaugt. Eine vorherige Instillation von Kochsalzlösung ist nicht erforderlich.

\section{Geschüitzte Bürste}

Die geschützte Bürste ist ein Doppellumen-Katheter, der an seinem distalen Ende mit einem Paraffin-Pfropf verschlossen ist. Die geschützte Bürste wird bis vor das zu untersuchende Bronchialostium platziert, anschließend wird der innere Katheter in das Ostium vorgeschoben und die sterile Bürste ausgefahren. Die Entfernung erfolgt in umgekehrter Reihenfolge. Die Bürste wird anschließend mit einer sterilen Schere abgeschnitten und in ein Transportmedium eingebracht (10).

\section{Bronchoalvoläre Lavage (BAL)}

Das größte Problem bei der BAL ist die Kontamination des Bronchoskopie-Arbeitskanals durch Keime der Mundhöhle. Grundsätzlich kann die BAL ohne speziellen Katheter durchgeführt werden, wenn: 1. der Zugang zum Lavage-Gebiet durch Katheterabsaugung gereinigt wurde, 2. keine Saugung durch den Arbeitskanal erfolgte und 3. Lokalanästhetika vermieden werden (sind in der Kultur wachstumshemmend). Nach Positionierung des Bronchoskops im betroffenen Lungenabschnitt erfolgt das fraktionierte Einspülen von Kochsalzlösung, die dann portionsweise aspiriert werden kann. Das Spülvolumen sollte so gewählt werden, dass $50 \mathrm{ml}$ Flüssigkeit zurückgewonnen werden, $100 \mathrm{ml}$ aber nicht überschritten werden. Die erste gewonnene Portion wird bei dieser Methode verworfen.

\section{kurzgefasst: Die klinische Diagnose einer nosokomialen Pneumonie unter Respirator-Therapie umfasst ein neues oder persistierendes Infiltrat in der Röntgenaufnahme des Thorax zwei der drei folgenden Kriterien: 1. Leuko- zytose oder Leukopenie, 2. Fieber oder Hypothermie und 3. prurulentes Bronchialsekret. Ein Erregernachweis (möglichst vor Beginn der antibiotischen Therapie) soll- te angestrebt werden.}

Ein Algorithmus zum empfohlenen Vorgehen bei der Diagnostik der nosokomialen Pneumonie findet sich in Abb.1.

\section{Literatur}

1 Rüden H, Gastmeier P, Wischnewski N, Hauer T, Schlingmann J, Schumacher M, Daschner F. Prävalenz der wichtigsten nosokomialen Infektionen in Deutschland. Ergebnis der NIDEP-Studie. Bundesgesundheitsblatt 1997; 6

2 Steinbrecher E, Sohr D, Nassauer A, Daschner F, Rüden H, Gastmeier P. Die häufigsten Erreger bei Intensivpatienten mit nosokomialen Infektionen. Ergebnisse des Krankenhaus-Infektions-Surveillance-Systems (KISS). Chemotherapie Journal 2000; 5: 179-118

3 Geffers C, Koch J, Sohr D, Nassauer A, Daschner F, Rüden H, Gastmeier P. Aufbau einer Referenzdatenbank für nosokomiale Infektionen auf Intensivstationen. Anaesthesist 2000; 49: 732-737

4 George DC, Falk PS, Wunderink RG, Jr. Leeper KV, Meduri GU, Steere EL, Carbett CE, Mayhall CG. Epidemiology of ventilator acquired pneumonia based on protected bronchoscopic sampling. Am J Respir Crit Care Med 1998; 158: $1839-1847$

Unertl K, Heininger A. Prävention der nosokomialen Pneumonie: Mitteilung der Komission für Krankenhaushygiene und Infektionsprävention am Robert Koch-Institut. Bundesgesundheitsbl - Gesundheitsforsch - Gesundheitsschutz. 2000; 43: 302-309

6 Bodmann KF, Lorenz J, Bauer TT, Ewig S, Trautmann M, Vogel F. Nosokomiale Pneumonie. Prävention, Diagnostik und Therapie. Ein Konsensuspapier der PEG und der DGP. Chemotherapie Journal 2002; in press

7 Fagon JY, Cluske J, Hance HJ, Guiguet M, Trouillet JC, Domart Y, Pierre J, Gibert C. Detection of nosocomial lung infection in ventilated patients. Use of a protected specimen brush and quantitative culture techniques in $147 \mathrm{pa}$ tients. Am Rev Respir Dis 1998; 138: 110-116

8 Sanchez-Nieto JM, Torres A, Garcia-Cordoba F, El-Ebiary M, Carillo A, Ruiz J, Nunez ML, Niederman M. Impact of invasive and noninvasive quantitative culture sampling on outcome of ventilator associated pneumonia: a pilot study. Am J Respir Crit Care Med 1998; 157: 371-376

9 Li J, Plorde JJ, Carlson LG. Effects of volume and periodicity on blood cultures. J Clin Microbiol 1994; 32: 2829-2831

10 Ewig S, Dalhoff K, Lorenz J, Mauch H, Schaberg T, Ukena D, Welte T, Wilkens H, Witt C. Empfehlungen zur Diagnostik der nosokomialen Pneumonie. Pneumologie 1999; 53: 499-510 